\title{
Solvable $\mathcal{P} \mathcal{T}$ symmetric Hamiltonians
}

\author{
Miloslav Znojil \\ Ústav jaderné fyziky AV ČR, 25068 Řež, Czech Republic \\ e-mail: znojil@ujf.cas.cz, November 2, 2018
}

\begin{abstract}
Within the so called $\mathcal{P} \mathcal{T}$ symmetric version of quantum mechanics a brief review of the exactly solvable models is given. Distinction is made between the curved and straight coordinate lines, between their unbounded (aperiodic) and bounded (periodic) choices, and between the completely and partially solvable cases.
\end{abstract}

\section{[KEYWORDS $]$}

[ $\mathcal{P} \mathcal{T}$ symmetry, Schrödinger equation in complex domain, exact solutions]

\section{[AMS 1991 Mathematics Subject Classification]}

81P10 81Q05 81R40 47B50 34A20 15A57 


\section{Introduction}

$\mathcal{P} \mathcal{T}$ symmetric quantum mechanics [1] has independently been proposed and used as a methodical laboratory in quantum physics by several groups of authors: By Caliceti et al [2] in perturbation theory, by Bessis et al [3] in field theory and by Andrianov et al [4] in supersymmetric context.

This short review will pay attention to the (partially as well as completely) exactly solvable models within this framework, with emphasis on the results obtained by the present author.

\section{Complete solvability on curved paths}

One of the first exactly and completely solvable examples of a $\mathcal{P} \mathcal{T}$ symmetric system has been found by Cannata et al [4] and re-discovered by Bender et al [5] more than one year later. Its modified Schrödinger bound state problem is defined on certain curved, left-right symmetric "generalized coordinate" lines in the complex plane. Mathematically, it is defined via exponential potential and proves exactly solvable in terms of Bessel functions. Due to its relationship to a power-law forces in the large exponent limit, it can be most simply interpreted as a certain smooth and non-Hermitian $\mathcal{P} \mathcal{T}$ symmetric analogue of the current square well.

Recently, a double-well counterpart of the latter set of models has been shown exactly solvable, in terms of Laguerre polynomials, in ref. [6]. In a way similar to the above "single well" example its paths of integration are the same, curved complex lines again. Their spectra exhibit a puzzling and highly unexpected feature of certain coupling-dependent re-arrangements mediated by "unavoided" crossings at critical points. The phenomenon reflects the non-Hermiticity of the Hamiltonian [7]. 
Via a suitable Liouvillean change of the variables in the above double-well-like differential Schrödinger equation one can immediately obtain another Laguerre-related solvable system with the potential of the Coulombic single-pole form. In this case the deformation of the integration path plays the beneficial role of a natural regularization prescription. At the same time it also leads to the necessity of working with the complex charges in a way described in ref. [8]. Also the related spectrum of energies exhibits certain unexpected features: Positivity, a coexistence of the growth and decrease with the increasing coupling strength, etc.

A clear mathematical explanation of behaviour as well as a consistent and/or possible physical interpretation of the counterintuitive models of this type remain unclarified up to now. Much better situation emerges in the case of the integration curves defined as the left-right-symmetric straight lines.

\section{Straight paths}

Using the language of the so called Kustaanheimo - Steifel transformation (cf. [9] for detailed references) both the above curvilinear examples can be shown equivalent to the $\mathcal{P} \mathcal{T}$ symmetric harmonic oscillator of ref. [10 with a centrifugal term regularized by the mere downward complex shift of the (full) real axis,

$$
\left(-\frac{d^{2}}{d x^{2}}+x^{2}-2 i c x+\frac{\alpha^{2}-1 / 4}{(x-i c)^{2}}\right) \varphi(x)=\left(E+c^{2}\right) \varphi(x), \quad \varphi(x) \in L_{2}(-\infty, \infty) .
$$

This oscillator with the Laguerre-polynomial normalizable solutions

$$
\varphi_{( \pm n)}(x)=\mathcal{N} \cdot(x-i c)^{ \pm \alpha+1 / 2} e^{-(x-i c)^{2} / 2} L_{n}^{( \pm \alpha)}\left[(x-i c)^{2}\right], \quad n=0,1, \ldots
$$

possesses the non-equidistant spectrum of energies $E=E_{( \pm n)}=4 n+2 \pm 2 \alpha$ and represents a certain unperturbed limit of the quartic oscillator models of Buslaev and 
Grecchi [11]. These authors, unfortunately, did not notice the existence of the "quasieven", $(-n)$-signed half of the spectrum. This omission can be easily corrected. One just introduces a "two-to-one" isospectrality correspondence between the respective Hermitian and non-Hermitian anharmonic oscillator models of ref. [11].

Interpretation of the models "living" on the straight lines becomes significantly facilitated by the easier identification and interpretation of their complex components [12]. Immediate purely analytic constructions recover, e.g., the existence of models which are in a one-to-one correspondence to the so called shape-invariant real forces in one dimension (cf. their presentation in ref. [13) and on the half line (their $\mathcal{P} \mathcal{T}$ symmetric counterparts were described and listed in ref. [14]).

The situation is reviewed in ref. [15]. A fully general form of this type of analytic constructions dates back to the introduction of the so called Natanzon potentials and, in the present context, is thoroughly analyzed and described in ref. [16].

\section{Models with periodic boundary conditions}

A new and promising development of the $\mathcal{P} \mathcal{T}$ symmetric considerations has been recently inspired by the study of the two- and three-particle models [17]. The $\mathcal{P} \mathcal{T}$ symmetrization of the Hamiltonians has been again conjectured to be sufficient for keeping their spectrum real. The related "weakening of the Hermiticity" finds a natural generalization in the new context.

Particular attention has been paid to the possible non-Hermitian generalizations of the well known Calogero model [18]. In this setting the separability of the underlying partial differential Schrödinger equation in the hyperspherical coordinates helps us to reduce the problem to the "hyperangular" ordinary differential equation 
defined on a finite interval. In this way, in the simplest cases one has to solve the complexified ordinary differential equations of the generalized Pöschl-Teller type,

$$
\left(-\frac{d^{2}}{d \phi^{2}}+\frac{\ell(\ell+1)}{\sin ^{2} \phi}+\frac{\lambda(\lambda+1)}{\cos ^{2} \phi}\right) \chi(\phi)=E \chi(\phi)
$$

on an interval $\phi \in(-M \pi / 2, M \pi / 2)$ with a suitable integer $M$. These equations can be solved exactly in terms of the hypergeometric functions 19.

The strongly repulsive singularities at $\phi_{j}=j \pi / 2$ are currently not penetrable [20]. Here, they become regularized in a $\mathcal{P} \mathcal{T}$ symmetric manner which parallels a few older constructions on the unbounded intervals [14]. The quasi-symmetric and quasi-antisymmetric solutions arise from certain ad hoc boundary conditions [17, 21].

For the most elementary illustration let us now choose $\lambda=0$ and $M=2$. Then, the differential equation (2) possesses the two independent hypergeometric solutions,

$$
\chi^{( \pm)}(\phi)=(\sin \phi)_{2}^{1 / 2 \pm \alpha} F_{1}\left(u^{( \pm)}, v^{( \pm)} ; 1 \pm \alpha ; \sin ^{2} \phi\right), \quad \alpha=\ell+1 / 2>0
$$

where $2 u^{( \pm)}=1 / 2-\beta \pm \alpha$ and $2 v^{( \pm)}=1 / 2+\beta \pm \alpha$. On the boundary of convergence $\sin ^{2} \phi=1$ the matching of the logarithmic derivatives is equivalent to the termination of this series to the Gegenbauer polynomials,

$$
\chi(\phi)=\chi_{( \pm k)}(\phi)=(\sin \phi)^{1 / 2 \pm \alpha} C_{k}^{1 / 2 \pm \alpha}(\cos \phi), \quad k=0,1, \ldots
$$

The construction also quantizes the energies and gives them in the closed form,

$$
E=E_{( \pm k)}=(k \pm \alpha+1 / 2)^{2}, \quad k=0,1, \ldots
$$

This set of eigenvalues is composed of the two subsets in a way which resembles the above-mentioned non-equidistant spectrum of the $\mathcal{P} \mathcal{T}$ symmetrized singular harmonic oscillator (11) [10]. 


\section{Conclusions and outlook}

Historically, one of the first persuasive manifestations of the merits and power of the $\mathcal{P} \mathcal{T}$ symmetry has been offered by Bender and Boettcher 22] who discovered the quasi-exact (i.e., incomplete) solvability of the most common and popular quartic polynomial oscillators.

The plausible reasons of the unexpected delay of such an "obvious" observation are closely related to the above-mentioned "forgotten" energies. One has to keep in mind the "spontaneous" regularity of the singular potentials within the new formalism. This observation was made explicit in our paper [23] where the presence of the two additional singular terms has still been shown compatible with the quasi-exact solvability of quartic potentials.

In the quasi-exact context the changes of variables can play the same role as in the completely solvable models. This has been illustrated by the particular constructions of the decadic model [24] and of the harmonic + Coulomb superposition [25]. Further work in this direction is in progress [26].

\section{Acknowledgements}

Research assisted by the Grant Agency of the Academy of Sciences of the Czech Republic, contract Nr. A 1048004.

\section{References}

[1] C. M. Bender, S. Boettcher, K. A. Milton and P. N. Meisinger, J. Math. Phys. 40 (1999) 2201. 
[2] E. Calicetti, S. Graffi and M. Maioli, Commun. Math. Phys. 75 (1980) 51;

G. Alvarez, J. Phys. A: Math. Gen. 27 (1995) 4589;

M. F. Fernndez, R. Guardiola, J. Ros and M. Znojil, J. Phys. A 31 (1998) 10105.

[3] D. Bessis and J. Zinn-Justin, private communication (1992);

C. M. Bender and A. Turbiner, Phys. Lett. A 173 (1993) 442;

C. M. Bender and K. A. Milton, Phys. Rev. D 55 (1997) R3255;

C. M. Bender and S. Boettcher, Phys. Rev. Lett. 24 (1998) 5243;

E. Delabaere and F. Pham, Phys. Lett. A 250 (1998) 25 and 29.

[4] A. A. Andrianov, F. Cannata, J.-P. Dedonder and M. V. Ioffe, Int. J. Mod. Phys. A 14 (1999) 2675;

F. Cannata, G. Junker and J. Trost, Phys. Lett A 246 (1998) 219;

B. Bagchi and R. Roychoudhury, J. Phys. A 33 (2000) L1;

M. Znojil, F. Cannata, B. Bagchi and R. Roychoudhury, Phys. Lett. B 483 (2000) 284 .

[5] C. M. Bender, S. Boettcher, H. F. Jones and Van M. Savage, J. Phys. A 32 (1999) 6771.

[6] M. Znojil, Phys. Lett. A 264 (1999) 108.

[7] I. Herbst, private communication.

[8] M. Znojil and G. Levai, Phys. Lett. A 271 (2000) 327.

[9] P. Kustaanheimo and E. Steifel, J. Reine Angew. Math. 218 (1965) 204;

E. G. Kalnins, W. Miller Jr. and G. S. Pogosyan, arXiv: quant-ph/9906055.

[10] M. Znojil, Phys. Lett. A 259 (1999) 220. 
[11] V. Buslaev and V. Grecchi, J. Phys. A 36 (1993) 5541.

[12] M. Znojil, J. Phys. A: Math. Gen. 32 (1999) 7419;

M. F. Fernndez, R. Guardiola, J. Ros and M. Znojil, J. Phys. A 32 (1999) 3105.

[13] M. Znojil, J. Phys. A: Math. Gen. 33 (2000) L61.

[14] M. Znojil, J. Phys. A: Math. Gen. 33 (2000) 4561.

[15] M. Znojil, Rendiconti del Circ. Mat. di Palermo, to appear.

[16] G. Levai and M. Znojil, J. Phys. A: Math. Gen. 33 (2000) 7165.

[17] M. Znojil and M. Tater, Complex Calogero model with real energies, arXiv: quant-ph/0010087, submitted to J. Phys. A.

[18] F. Calogero, J. Math. Phys. 10 (1969) 2191;

A. Turbiner, Mod. Phys. Lett. A 13 (1998) 1473 with further references.

[19] F. Cooper, A. Khare and U. Sukhatme, Phys. Rep. 251 (1995) 267.

[20] S. Flügge, Practical Quantum Mechanics I (Springer, Berlin, 1971), p 89.

[21] M. Znojil, Phys. Rev. A 61 (2000) 066101.

[22] C. M. Bender and S. Boettcher, J. Phys. A 31 (1998) L273.

[23] M. Znojil, J. Phys. A 33 (2000) 4203.

[24] M. Znojil, J. Phys. A: Math. Gen. 33 (2000) 6825.

[25] M. Znojil, J. Phys. A: Math. Gen. 32 (1999) 4563.

[26] F. Gemperle, M. Znojil and F. Cannata, in preparation. 